\title{
Chemical NanoSensors and Microsystems for Air Pollution Detection
}

\author{
J.R. Morante ${ }^{1,2}$ \\ ${ }^{1}$ Departament d'Electrònica, Universitat de Barcelona, Spain \\ ${ }^{2}$ Institut de Recerca en Energia de Catalunya (IREC), Spain \\ jirmorante@irec.cat
}

\begin{abstract}
:
The understanding chemical to electrical transduction mechanisms at the nano scale level becomes a fundamental issue for developing and designing feasible and reliable gas sensors. Air pollution detection requires the simultaneous detection of oxidizing and reducing gases in the presence of many potential interfering molecules, especially water, under stable conditions and avoiding sensor degradation. In this contribution, on the base of the analysis performed on individual nanowire platforms, the transduction mechanisms at the nano scale level and associated kinetics are revised considering the influence of the temperature and illumination as well as the consequences of the pulsed or steady working modes on the signal threshold, selectivity and stability of the conductometric solid state based gas sensors. Potential integration of multiple sensors as multifunctional systems is also discussed and implemented including filters, pre-concentrators in several configurations including that of the integrated gas chromatography.
\end{abstract}

Key words: nanosensors, air pollution detection, gas sensors,

\begin{abstract}
Introduction
Air pollution detection is a big challenge as low thresholds, high level of selectivity and large stability are required [1]. Although different technologies for sensing can be applied, at the present contribution, the analysis is focused only on conductometric solid state based gas sensor elements and their potential integration in multifunctional systems[2].
\end{abstract}

Since the first works on the potential application of metal oxides as gas sensing materials, many effort have been done. However, in spite of the hard performed actions, still there are many unknown processes and reliable and feasible low cost and mass production of solid state gas sensors exist scarcely as extended commercial products. There is a large list of gas molecules to be measured but for centering our analysis significant reducing gas like $\mathrm{CO}$, oxidizing one like $\mathrm{NO}_{2}$ with one interfering molecule like $\mathrm{H}_{2} \mathrm{O}$ are, mainly, retained as representative target molecules.

Up to now, thin or thick films of nanocrystalline metal oxides have been used as sensing elements implemented as layers or beads in combination with a heater allocated in the supporting substrate or in the own bead. The evolution of the used technological strategies has moved from alumina substrate, consuming hundred of $\mathrm{mW}$ to micromachined substrate requiring only a few tens of $\mathrm{mW}$ for heating the sensing materials up to $400^{\circ} \mathrm{C}$. Moreover, the low thermal inertia presented for this micromachined system gives the possibility to work in a pulsed mode avoiding the steady state working mode and facilitating the excitation and detection kinetics. On the other hand, unlike to the usual laboratory test conditions that apply gas pulsed on the sensors, the low thermal inertia systems allow applying the heating pulse only during the measurement moment as well as the intensity of this heating pulse allow to determine the heating temperature adapted to the detection of the target molecules. These promising advantages have launched many new efforts for integrate such sensors as element in a multifunctional system $[3,4,5]$.

However, in spite of these improvements at the sensor device level, there are many missing on the details of the chemical to electrical transduction mechanisms taking place at the surface of the metal oxides. It is accepted that smaller gas sensor are giving higher signal level but any information is available on the 
response and recovery time constant for example. Likewise, there is also scarce information about the influence of the porosity degree or the influence of the layer thickness or, even, the role of the contact interface or about the material degradation processes originated themselves for the own reactivity with the gas molecules. Serious baseline drifts and signal variations when wearing out the sensor are found related with the influence of the surface characteristics on the transduction mechanisms. It introduces clear and significant limits in the application of the solid state gas sensors for air pollution detection. Furthermore, interfering gases is still a not solved feature and nowadays, humidity interferences are a pendent matter.

In this contribution, the basic chemical to electrical transduction mechanisms on the base of individual metal oxide nanowires platforms operated with the self-heating for reducing and oxidizing gases are reviewed. Likewise, their interferences with humidity are considered. The requirements for feasibility and reliability of integrated gas system for air pollution detection are also discussed.

\section{Experimental details}

Gas sensor platforms based on individual $\mathrm{SnO}_{2}$ nanowires [3-5] were fabricated by means of Focused Ion Beam lithography [6]. In order to control the self-heating effect, currenttemperature calibration curves where determined for each device under test following a procedure described elsewhere [3-5].

\section{Results on individual nanowire platforms:}

Many attempts have been performed to determine the time constant associated with the interaction of $\mathrm{CO}$, reducing gas, and $\mathrm{NO}_{2}$, oxidizing gas obtaining a wide range of values and dependence with the thicknesses of the layer[7,8]. Our experimental platforms allow to have already the gas in ambient avoiding gas injection and gas diffusion times. Only the heating time inertia is putting limitations for having straightforward the associated response and recovery time constants. In figure 1 , the thermal inertia associated constant times are shown which are not too much different of that
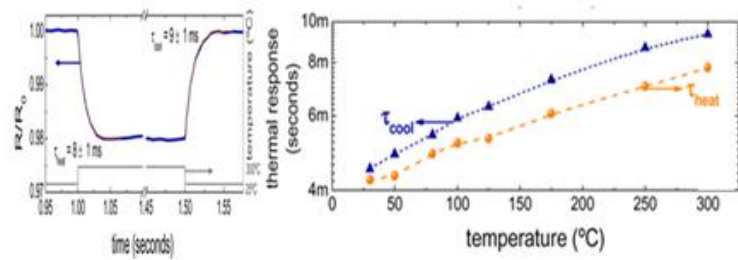

Fig.1: Thermal inertia constant time determined for individual nanowire platforms presented by the micromachined substrates. In figure 2, the direct kinetic constant times for $\mathrm{CO}$ and $\mathrm{NO}_{2}$ are shown as a function of the temperature.
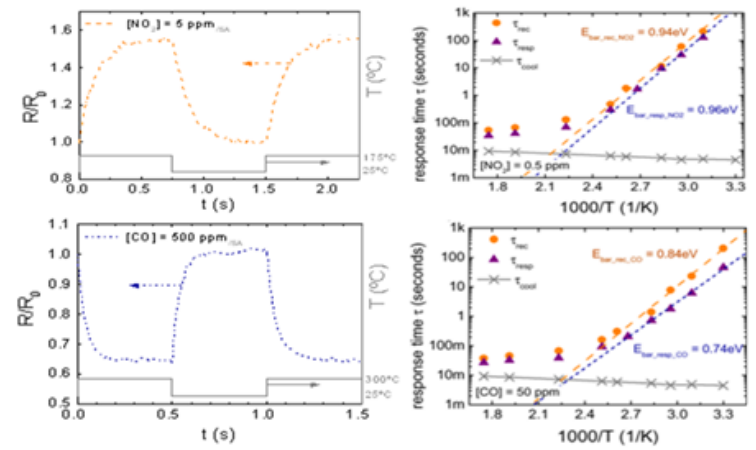

Figure 2 Constant times associated to the kinetic sensing reactions on $\mathrm{SnO}_{2}$

These values give the time limits response for all the sensors. Figure 3 shows the comparison with previous reported values.

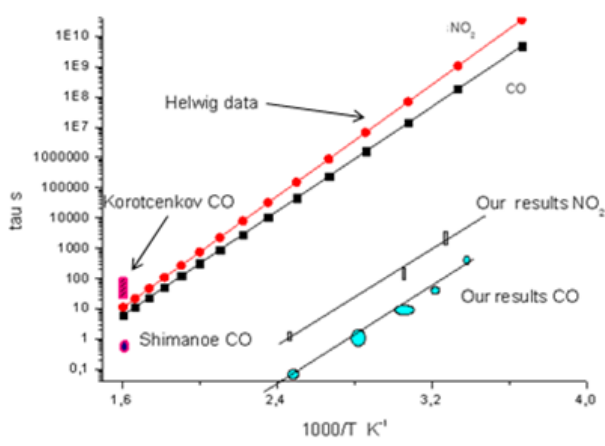

Figure 3 Time constants versus temperature

The time constant is specific for each molecule reaction taking place on the metal oxide surface and therefore as it can be determined; it can offer a methodology to identify competing processes happens simultaneously on the surface and altering the electrical parameters like it occurs with the interfering gases. In figure 4 , the variations of the CO time constant due to their competition with the water molecules is plotted as a function of the relative humidity.

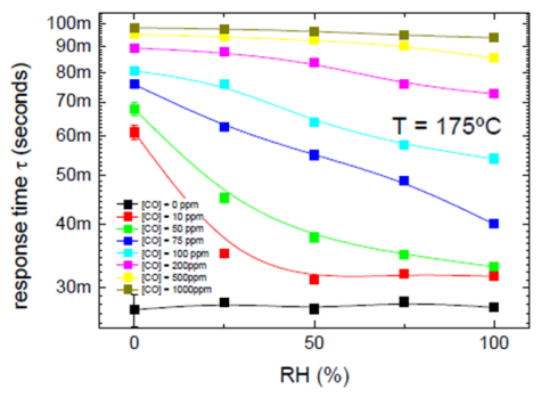

Fig 4: Influence of the competing mechanism due to the presence of water on the CO time constant

The mathematical analysis of the obtained transients, allow knowing the pure time 
constant to fit the transients using two transients with amplitudes and time constant associated to the interaction of $\mathrm{CO}$ and with $\mathrm{H}_{2} \mathrm{O}$ respectively [9]. See figure 5 .

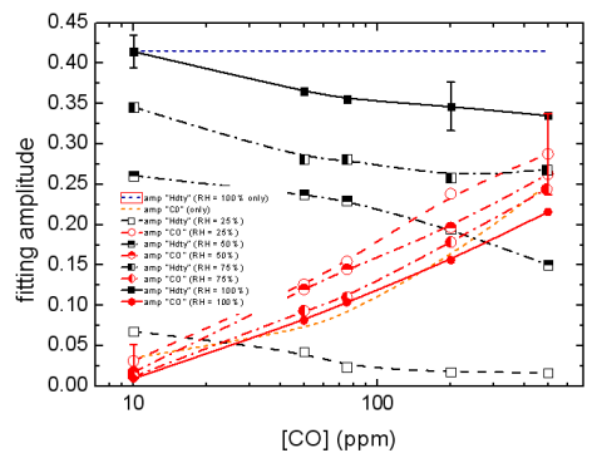

Fig. 5: Fitted amplitudes for each contribution

These results explain the behavior found for the usual plot of the logarithm of the sensor response versus the concentration logarithm reported in figure 6 .

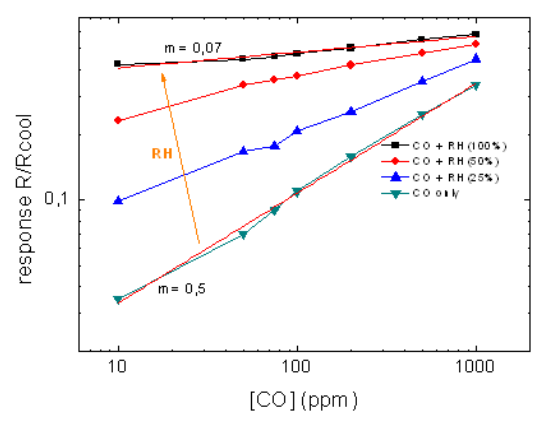

Fig. 6 Log-Log Plot for CO with humidity

So, the transient signal analysis opens an outstanding new option for discriminating interfering gases and achieving a better selectivity that increases the sensor feasibility and reliability for air pollution measurements. This procedure can also be applied to other target gases if their transduction mechanisms are deepen analyzed versus the temperature.

Alternatively, for some other gases like oxidizing $\mathrm{NO}_{2}$, the sensing reactions taking place at the surface can be analyzed as a function of the illumination of selected photon energy and flux density for discriminating on their contribution on the transient of the sensor electrical signal. The found behavior in comparison with other gases becomes a fingerprint of the target gas and gives us support for achieving also a better selectivity against interfering gases. This methodology can also be used as checking of the surface state[10-11].

\section{Chemical gas integrated systems:}

Micromechanized membranes and other more complex hotplates or gas sensor platforms can easily be integrated on the base of silicon micro technology including all the features for working with pulsed mode and treatments [12-14] fig.8.

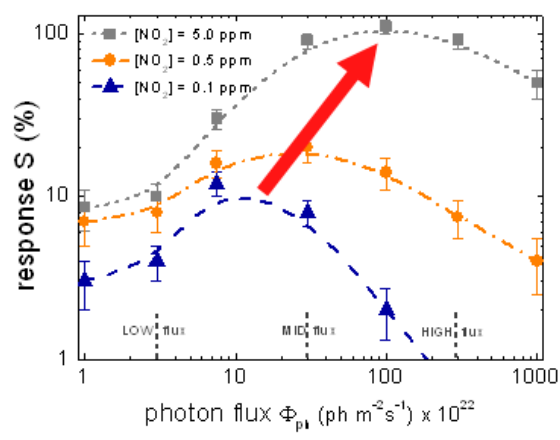

Fig. 7 Sensor response versus photon flux for $\mathrm{NO}_{2}$. Dashed lines correspond to theoretical fittings.

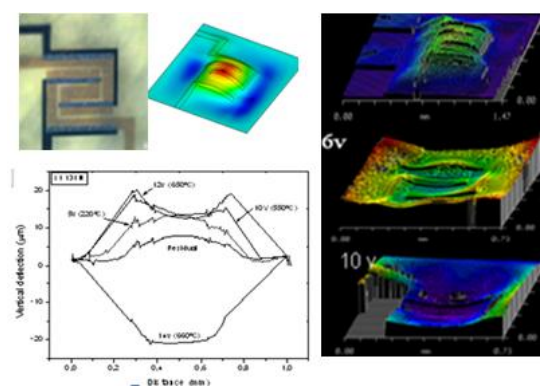

Fig.8 Improved micromechanized integrated gas sensing platforms

Nowadays, improved integration micro technologies, like SOI based approach, facilitates advanced multifunctional design and gas system implementation. Integrated systems requires of a multisensor advanced platforms in order to include not only the above described working functions and signal processing but the possibility to combine multiple sensor at the same devices with a minimized power consumption, communication issues and an adequate packaging. It gives many powerful options: i) the use of active filters for avoiding some interfering gases o poisoning elements; ii) the use of pre concentrators; iii) the implementation of serial or parallel fluidic circuits for exciting integrated gas sensors sequentially or simultaneously but using independent chambers;

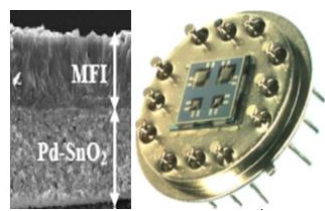

Fig. 9 Multisensor systems including a zeolyte filter onto the sensing layer.

iv) the implementation of new strategies for enhancing the gas sensing capabilities as for example the implementation of sensors as 
ending sensors of a chromatographic integrated column that contribute to separate mixed gases and, hence, increasing selectivity; v) the combined integration with other functional sensors, actuators and functions like temperature, humidity, pressure, flow ....sensors or accompanying harvesting systems to address the integrated system toward full autonomous systems [15].

\section{Conclusion}

The improved understanding and knowledge of the chemical to electrical transduction mechanisms at the nano scale level is essential for an adequate design and optimization of multifunctional integrated gas sensor systems. The combined use of filters, preconcentrators, time resolved gas separation together with the powerful capability for applying working modes adapted to the transduction mechanisms in order to increase selectivity and stability, become fundamental milestones for achieving reliable and feasible integrated gas sensors for air pollution detection avoiding unwanted interfering gases effects and keeping suitable threshold of the required environment gases.

\section{Acknowledgements:}

The research leading to these results has received funding from the European Community's $7^{\text {th }}$ Framework Program under grant agreement no. 247768 (S3) no. 288481 (SOI-HIT) and from the COST program 2011-19706 (EuNetAir)

\section{References}

[1] http://www.epa.gov/oar/airpollutants.html

[2] J Puigcorbé, D Vogel, B Michel, A Vilà, I Gràcia, C Cané and J R Morante. Thermal and mechanical analysis of micromachined gas sensors Journal of Micromechanics and microengineering 13 (5) 548-556 (2003)

[3] F. Hernandez-Ramirez, J.D. Prades, A. Tarancon, S. Barth, O. Casals, R. Jimenez-Diaz, E. Pellicer, J. Rodriguez, M.A. Juli, A. RomanoRodriguez, J.R. Morante, S. Mathur, A. Helwig, J. Spannhake, G. Mueller, Portable microsensors based on individual $\mathrm{SnO} 2$ nanowires, Nanotechnology 18, 495501(2007)

[4] J.D. Prades, R. Jimenez-Diaz, F. HernandezRamirez, S. Barth, A. Cirera, A. RomanoRodriguez, S. Mathur, J.R. Morante, Ultralow power consumption gas sensors based on selfheated individual nanowires, Applied Physics Letters 93, 123110 (2008).

[5] J.D. Prades, R. Jimenez-Diaz, F. HernandezRamirez, A. Cirera, A. Romano-Rodriguez, J.R. Morante, Harnessing self-heating in nanowires for energy efficient, fully autonomous and ultrafast gas sensors, Sensors and Actuators B Chemical 144, 1-5 (2010).
[6] F. Hernandez-Ramirez, A. Tarancon, O. Casals, E. Pellicer, J. Rodriguez, A. Romano-Rodriguez, J.R. Morante, S. Barth, S. Mathur, Electrical properties of individual tin oxide nanowires contacted to platinum electrodes, Physical Review B 76, 085429 (2007)

[7] J. D. Prades, F. Hernández-Ramírez, T. Fischer, M. Hoffmann, R. Müller, N. López, S. Mathur, and J. R. Morante Quantitative analysis of COhumidity gas mixtures with self-heated nanowires operated in pulsed mode Applied Physics Letters 97 (24) 243105 (2010)

[8] F. Hernandez-amirez, J.D.Prades, A. Tarancon, S.Barth, O.Casals, R.Jimenez-Diaz, E. Pellicer, J.Rodriguez, J.R.Morante,S. Mathur, A. Romano-Rodríguez.Insight into the Role of Oxygen Diffusion in the Sensing Mechanisms of SnO2 Nanowires. Advanced Functional Materials, 18 (19) 2990 (2008)

[9] J. D. Prades, R. Jimenez-Diaz, F. HernandezRamirez, J. Pan, A. Romano-Rodriguez, S. Mathur, and J. R. Morant ${ }^{\mathrm{e}}$, Direct observation of the gas-surface interaction kinetics in nanowires through pulsed self-heating assisted conductometric measurements_Applied Physics Letters 95 (5) 053101 (2009)

[10] J.D. Prades, R. Jimenez-Diaz, M.Manzanares F. Hernandez-Ramirez , A. Cirera , A. RomanoRodriguez , S. Mathur and J. R. Morante. A model for the response towards oxidizing gases of photoactivated sensors based on individual SnO2 nanowires. Physical Chemistry Chemistry Physical 11 (46) 10881-10889 (2009

[11] F. Hernandez-amirez, J.D.Prades, A. Tarancon, S.Barth, O.Casals, R.Jimenez-Diaz, E. Pellicer, J.Rodriguez, J.R.Morante,S. Mathur, A. Romano-Rodríguez.Insight into the Role of Oxygen Diffusion in the Sensing Mechanisms of SnO2 Nanowires. Advanced Functional Materials, 18 (19) 2990 (2008)

[12] Puigcorbe J; Vila A; Morante JR Thermal fatigue modeling of micromachined gas sensors Sensors and Actuators 95 (1-3) 275-281, (2003)

[13] J. Cerda Belmonte J. Manzano, J. Arbiol, A. Cirera, J. Puigcorbe, A. Vila,N. Sabate, I. Gracia, C. Cane, J.R. Morante Micromachined twin gas sensor for $\mathrm{CO}$ and O-2 quantification based on catalytically modified nano-SnO2 Sensors and Actuators B-Chemical 114, (2) 881-892 (2006)

[14] J. Cerda Belmonte , J. Puigcorbe , J. Arbiol , A. Vila , J.R. Morante ,N. Sabate , I. Gracia , C. Cane High-temperature low-power performing micromachined suspended micro-hotplate for gas sensing applications Sensors and Actuators B-Chemical 114,( 2) 826-835 (2006)

[15] F. Hernandez-Ramirez , J. D.Prades , R. Jimenez-Diaz , T. Fischer , A. RomanoRodriguez, S. Mathur and J. R. Morante On the role of individual metal oxide nanowires in the scaling down of chemical sensors_.Physical chemistry chemical physics 11 ( 33) 7105 (2009) 